\title{
Nonsense Suppression Therapy: An Emerging Treatment for Hereditary Skin Diseases
}

\author{
Jiangfan $\mathrm{YU}^{1,2}$, Bingsi TANG ${ }^{1,2}$, Xinglan $\mathrm{HE}^{1,2}$, Puyu ZOU ${ }^{1,2}$, Zhuotong ZENG ${ }^{1,2}$ and Rong XIAO ${ }^{1,2}$ \\ ${ }^{1}$ Department of Dermatology and 'Hunan Key Laboratory of Medical Epigenetics, Second Xiangya Hospital, Central South University, \\ Changsha, China
}

\begin{abstract}
Nonsense mutations cause the premature termination of protein translation via premature termination codons (PTCs), leading to the synthesis of incomplete functional proteins and causing large numbers of genetic disorders. The emergence of nonsense suppression therapy is considered to be an effective method for the treatment of hereditary diseases, but its application in hereditary skin diseases is relatively limited. This review summarizes the current research status of nonsense suppression therapy for hereditary skin diseases, and discusses the potential opportunities and challenges of applying new technologies related to nonsense suppression therapy to dermatology. Further research is needed into the possible use of nonsense suppression therapy as a strategy for the safer and specific treatment of hereditary skin diseases.
\end{abstract}

Key words: readthrough; aminoglycosides; PTC124; nonsensemediated decay; suppressor tRNA; hereditary skin diseases.

Accepted Dec 2, 2021; Epub ahead of print Jan 27, 2022

Acta Derm Venereol 2022; 102: adv00658.

DOI: $10.2340 / a c t a d v . v 102.353$

Corr: Zhuotong Zeng and Rong Xiao, Department of Dermatology, Second Xiangya Hospital of Central South University, Changsha 410011, China. E-mail: zengzhuotong@csu.edu.cn, xiaorong65@csu.edu.cn

$\mathrm{A}$ lmost all organisms use the same genetic code, consisting of 61 sense codons that code amino acids and 3 nonsense (or stop) codons that do not encode any amino acids. The process of protein translation begins with the recognition of the initiation codon and ends with the recognition of a stop codon, and involves the participation of mRNA, rRNA, tRNA, ribosomes, and a series of protein factors (1). A nonsense mutation refers to the mutation of a codon encoding a certain amino acid into a stop codon due to substitution of bases. A premature termination codon (PTC) has the same structure and function as a stop codon in the normal translation process, leading to premature termination of protein translation, resulting in an incomplete non-functional or harmful polypeptide chain (2). PTCs can trigger nonsense-mediated mRNA decay (NMD), which targets mRNAs containing PTCs for degradation, thereby preventing their translation and further reducing protein levels (3). Genetic diseases caused by nonsense mutations account for approximately $12 \%$ of all genetic diseases (4). In view of this high incidence, corresponding nonsense suppression

\section{SIGNIFICANCE}

Nonsense suppression therapy refers to an effective means of preventing protein translation termination and ultimately alleviating disease symptoms by promoting PTCreadthrough. For decades, this therapy has been successfully used in the clinical treatment of certain genetic diseases, but its attempts in hereditary skin diseases are still shallow. Here, we review the research status of the latest nonsense suppression treatments for hereditary skin diseases, and describes the readthrough mechanisms of classic aminoglycosides, the novel compound PTC124, NMD inhibitors, and suppressor tRNA. We also present future prospects for topical formulations, combination drugs, new compounds, emerging technologies in dermatology related to nonsense suppression therapy.

therapy has great potential for therapeutic applications, which is a strategy aimed at restoring protein functional defects, reversing disease phenotypes, and improving the progression of genetic diseases.

Nonsense suppression treatments typically prevent the termination of protein translation via the PTC-readthrough. Specifically, there are various methods, such as classic aminoglycosides, NMD inhibitors, suppressor tRNA, RNA pseudouridylation, RNA editing, and the CRISPR/Cas9 system $(3,5)$. In eukaryotes, the termination process of protein translation involves 2 types of release factors (RF), eRF1 and eRF3. At the ribosome A site, eRF1 directly recognizes and interacts with 3 stop codons, resulting in the release of the peptide chain. The combination of eRF3 and eRF1 promotes translation termination activity, then tRNA, mRNA, and ribosomal subunits are dissociated (6) (Fig. 1). When eRF1 directly recognizes and binds to PTCs at the ribosome A site, if the adjacent homologous tRNA competes with eRF1 for binding to the PTC, the amino acid transported by the homologous tRNA will replace the original stop codon. Thus, protein translation can continue to form proteins of normal length or of similar functions, which is called PTC-readthrough. As long as there is no substitution of the necessary amino acid, or even when it contains a wrongly incorporated amino acid, the protein still has part of its normal activity.

As early as 1982, Temple et al. (7) proposed that, through specific anticodon mutations, a suppressor tRNA can inhibit nonsense mutations in $\beta$-thalassemia 


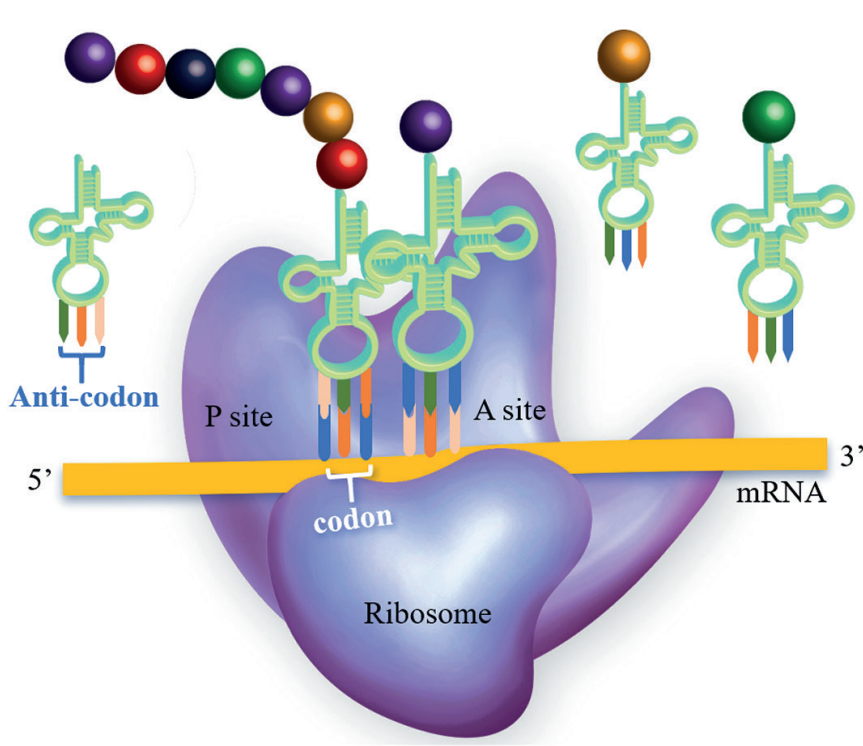

Normal translation

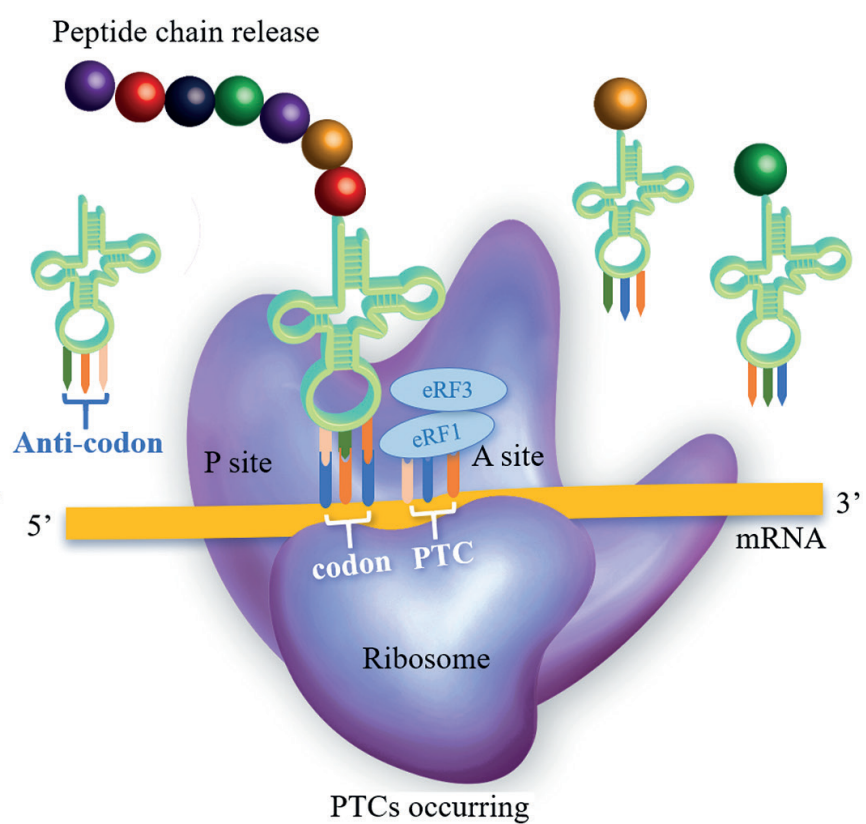

PTCs occurring

Fig. 1. Normal translation of proteins (left) and termination of protein translation when a premature termination codon (PTC) occurs (right).

mRNA and also provide a method for gene therapy of $\beta$-thalassemia caused by nonsense mutations. Similarly, Kiselev et al. (8) reported that the use of suppressor tRNA can produce dystrophin, and some Duchenne muscular dystrophy (DMD) cases are caused by nonsense mutations in the dystrophin gene. Howard et al. reported that treatment with aminoglycoside antibiotics can inhibit nonsense mutations in severe cystic fibrosis (CF) and promote the expression of functional proteins (9). With continuing research, nonsense suppression has gradually found applications in treating multiple disease types, such as thrombotic diseases, nervous system diseases, ocular diseases, and hereditary skin diseases (10-27) (Table I). For some recessive disorders, successful gene

Table I. Nonsense mutation and nonsense suppression studies in other diseases

\begin{tabular}{|c|c|c|c|c|c|}
\hline Disease & Gene & Mutation & Stop codon & Nonsense suppression therapy & Reference \\
\hline $\mathrm{CF}$ & CFTR & G542X & UGA G & Gentamicin & $(10)$ \\
\hline DMD & $D M D$ & $m d x$ & UAA A & Gentamicin & $(11)$ \\
\hline SMA & SMN1 & W102X & UAG T & Geneticin & $(12)$ \\
\hline Menkes syndrome & ATP7A & R201X & UGA & Copper & (13) \\
\hline A-T & ATM & $\begin{array}{l}\text { TAT51 } \\
\text { AT153LA } \\
\text { AT187LA } \\
\text { AT185LA }\end{array}$ & $\begin{array}{l}\text { UGA C } \\
\text { UGA A } \\
\text { UAA G } \\
\text { UAA G }\end{array}$ & Geneticin & $(14)$ \\
\hline CDKL5 syndrome & $C D K L 5$ & $\begin{array}{l}\text { R59X } \\
\text { R134X } \\
\text { Q347X } \\
\text { E364X }\end{array}$ & $\begin{array}{l}\text { UGA } \\
\text { UGA } \\
\text { UAG } \\
\text { UAA }\end{array}$ & Geneticin, gentamicin, PCT124 & $(15)$ \\
\hline Hurler syndrome & IDUA & $\begin{array}{l}\text { Q70X } \\
\text { W402X }\end{array}$ & $\begin{array}{l}\text { UAG C } \\
\text { UAG G }\end{array}$ & Gentamicin & $(16)$ \\
\hline Cystinosis & CTNS & $753 G \rightarrow A(W 138 X)$ & UGA & Gentamicin & $(17)$ \\
\hline XNDI & AVPR2 & E242X & UAG C & Geneticin & $(18)$ \\
\hline XLRP & $R P 2$ & Arg120stop & UGA G & Gentamicin & (19) \\
\hline \multirow[t]{2}{*}{ Choroideremia } & $\mathrm{CHM}$ & & & Gentamicin, paromomycin, PTC124, PTC-414 & $(20)$ \\
\hline & CHM, NOI, GUP & $\begin{array}{l}\text { Q32X } \\
\text { R139X } \\
\text { Q524X }\end{array}$ & $\begin{array}{l}\text { UAA } \\
\text { UGA } \\
\text { UAG }\end{array}$ & Gentamicin, paromomycin & $(21)$ \\
\hline USH1 & PCDH15 & $\begin{array}{l}\text { p.R3X } \\
\text { p.R643X } \\
\text { p.R929X } \\
\text { p.R245X }\end{array}$ & $\begin{array}{l}\text { UGA C } \\
\text { UGA G } \\
\text { UGA A } \\
\text { UGA A }\end{array}$ & Geneticin, paromomycin, gentamicin, NB30 & $(22)$ \\
\hline RCS & PAX2 & & & & $(23)$ \\
\hline CHARGE syndrome & $\mathrm{CDH7}$ & & & & $(24)$ \\
\hline HCS & NOTCH2 & The last coding exon & & & $(25)$ \\
\hline$\beta$-thalassemia & tRNA ${ }^{\text {Lys }}$ & & UAG & Suppressor tRNA & (7) \\
\hline VWD & $V W F$ & 2908del C in exon 22 & & & $(26)$ \\
\hline SDS & SBDS & $\mathrm{K} 62 \mathrm{X}$ & UGA & PCT124 & $(27)$ \\
\hline
\end{tabular}

SMA: spinal muscular atrophy; A-T: ataxia-telangiectasia; XNDI: X-linked nephrogenic diabetes insipidus; XLRP: X-linked retinitis pigmentosa; USH1: type 1 Usher syndrome; RCS: Renal coloboma syndrome; HCS: Hajdu-Cheney syndrome; VWD: Von Willebrand disease; SDS: Shwachman-Diamond Syndrome. 
therapy can be obtained by partial correction of translation because even a small amount of functional protein is sufficient to improve clinical symptoms (28).

In dermatology, previous clinical studies have shown that topical and systemic use of readthrough compounds can improve nonsense-mediated skin disease phenotypes. The aim of this paper is to review the mechanisms for controlling nonsense suppression, and its relevance to hereditary skin diseases, providing evidence for the application of nonsense suppression therapy as a viable treatment option for incurable hereditary skin diseases.

\section{NONSENSE SUPPRESSION THERAPY}

\section{Aminoglycosides}

Aminoglycosides are a kind of natural or semi-synthetic antibiotic. By directly targeting the bacterial ribosome A site to change its conformation, they lead to tRNA reading errors and interfere with protein synthesis (29). In eukaryotic cells, natural stop codons are surrounded by specific upstream and downstream sequences, and there are multiple cellular mechanisms that make it difficult to readthrough, with a probability of $0.001 \%-0.1 \%$ (30); however, PTCs usually lack the protection of these sequences, and aminoglycosides can promote the binding of nearly homologous tRNA to PTCs, replacing eRF1, allowing protein synthesis to continue, and suppressing nonsense mutations. Aminoglycosides have strong sequence specificity for PTC-readthrough therapy, which depends on the stop codon itself and the surrounding nucleotide sequence (31). Therefore, aminoglycosides act mainly on nonsense mutation sites (32). Even if the readthrough of the natural stop codon occurs, its effect is slight and will not have a significant impact.

Different aminoglycoside drugs have different nonsense mutation suppression activities (33). Different chemical structures determine the affinity of interactions with ribosomes and may influence the PTC-readthrough efficiency (34). Even different components of the same drug with very similar structures can have different nonsense mutation suppression activities. For example, gentamicin is a mixture of multiple compounds which have different nonsense mutation suppression activities. The main component is the $\mathrm{C}$ group, in addition to a small amount of gentamicin B, gentamicin B1 and so forth. Gentamicin B1 has the main nonsense mutation suppression activity, while the very close structural analog gentamicin B lacks readthrough activity. Thus, the amount of different ingredients of the same medicine may vary between formulations $(35,36)$. This explains why the same drug can have different therapeutic effects. Moreover, the core component, which has the main readthrough effect, can achieve an ideal nonsense suppression effect even at a low dose. Increasing the concentration of non-core components will not increase the readthrough activity or drug toxicity, but will reduce the readthrough activity of the core components $(35,37)$, suggesting that components with major nonsense mutation suppression activity may also have major drug toxicity.

Despite their proven clinical utility, these therapies also have many shortcomings, leading to potential sideeffects, including ototoxicity and nephrotoxicity, as well as increased drug resistance. In certain diseases caused by nonsense mutations, they can restore only part of the protein function, and in many diseases they cannot restore any protein function (38). In addition, the binding efficiency of different drugs is not the same, which may lead to the synthesis of full-length or truncated proteins, and the function of these proteins is not yet known and may be harmful (37).

The application of aminoglycosides has opened the door to the treatment of nonsense mutations. Although clinical applications are limited by their toxicity and the low readthrough efficiency of the stop codon, the benefits are beyond doubt. In order to reduce drug toxicity, aminoglycoside derivatives have been developed (39). They are less toxic and have fewer accompanying negative effects. Therefore, they may be more beneficial to suppress nonsense mutations, although this potential advantage requires further evaluation in vivo.

\section{Non-aminoglycosides}

Among the non-aminoglycoside drugs, the most promising drug at present is PTC124, also known as ataluren (brand name Translarna), which is a new type of small molecule drug that has been shown to promote nonsense suppression through high-throughput screening (40). It shares no structural similarity to aminoglycosides, and consequently, it lacks the associated toxic side-effects (41). It is more specific and selective for the PTCreadthrough without affecting the translation termination of natural stop codons (42). PTC-124 is tolerable orally and can be used at significantly lower dosages to obtain higher levels of readthrough compared with aminoglycosides (43). Its mechanism may be that it forms a stable complex with PTC-containing mRNA, preventing eRF1 from recognizing the PTC and inhibiting translation termination (44). Recent research shows that PTC124 is selective to the ribosomal A site and promotes the insertion of amino acids in the PTC site of homologous tRNAs to allow translation to continue (45). PTC124 has been approved for Phase 2 clinical trials for over 10 years and Phase 3 clinical trials for 5 years. It seems to have a positive effect on a variety of diseases, but has also been reported to be ineffective (46). This may be related to cell metabolism, drug solubility, compound permeability differences, the influence of other drugs, NMD levels, the significant difference in the activity of PCT124 in vivo and in vitro, or other factors (47). In addition to PTC124, there are many other small molecule compounds that can promote PTC-readthrough, such as RTC13, RTC14, GJ071, GJ072, RTC204, RTC219, 
BZ6, BZ16, and clitocine (48). These compounds display reduced toxicity and have varying degrees of nonsense suppression activity (49). Clitocine works at the transcriptional level and promotes the decoding of nonsense codons (50). However, the specific mechanism of action of other compounds is still unclear. Many non-aminoglycoside antibiotics have also been shown to suppress PTCs. Negamycin is an antibiotic whose chemical structure is different from aminoglycosides, but its effect on ribosomal decoding is similar (51). In addition, tylosin, josamycin, spiramycin, erythromycin, azithromycin, and other macrolide antibiotics also have a certain PTC-readthrough effect. They probably promote PTC-readthrough by inhibiting the release of the peptide chain from the ribosome (52).

\section{Suppression of nonsense-mediated $m R N A$ decay}

NMD preserves normal physiological levels of transcription and translation, and allows the body to dynamically adjust to different environments (53). It plays a role in recognizing and degrading PTC-containing mRNA, thereby preventing the translation of mutant transcripts and further reducing the level of abnormal proteins (54). By inhibiting NMD, mutated mRNA transcripts are stabilized, and the content of peptides is increased, thereby enhancing the readthrough effect to alleviate certain disease phenotypes. The efficiency of NMD may be related to sequences upstream and downstream of the PTC, or it may vary for different mutations and different genes (47). NMD suppression therapy may also have potentially harmful side-effects. Because NMD is active in many cellular processes, in addition to regulating the expression of transcripts involving nonsense mutations, NMD also regulates the expression of other gene transcripts. It affects DNA replication, repair, and telomere integrity during the cell cycle, and thus maintains the stability of the genome. Excessive suppression of NMD may bring some negative effects (55). Therefore, the reasonable choice of NMD inhibitors in treatment is worthy of consideration.

\section{Suppressor tRNA}

Suppressor tRNA refers to tRNA molecules with mutations in the anticodons that act by correcting nonsense mutations. They exist naturally and can also be obtained by editing the anticodons (56). Suppressor tRNA promotes amino acid substitutions of PTCs to generate full-length functional proteins and restore protein activity. The readthrough efficiency of suppressor tRNA will depend on its expression level and stability in target organs, the nature of amino acids substituted for nonsense codons, the contextual sequence of codons, the efficiency of translation bypass, and various cellular mechanisms $(5,7,57)$. The degradation rate of tRNA is slow, hence the action time is long. The chemical structure of suppressor tRNA is similar to that of natural tRNA, and it has little toxicity and should rarely cause an immune response. However, since it may also cause the readthrough of natural stop codons, and the expression varies with different diseases, further experiments are needed to evaluate its safety and effectiveness.

\section{NONSENSE SUPPRESSION IN HEREDITARY SKIN DISEASES}

Nonsense mutations have been shown to exist in some hereditary skin diseases, and the use of nonsense suppression therapy can be used to effectively treat them. Table II summarizes and lists the PTC-related hereditary skin diseases that have been studied.

\section{Pseudoxanthoma elastica}

Pseudoxanthoma elastica (PXE) is caused by mutations in the $A B C C 6$ gene that encodes the transmembrane transport protein ABCC6. Approximately $35 \%$ of $A B C C 6$

Table II. Nonsense mutation and nonsense suppression studies in hereditary skin diseases

\begin{tabular}{|c|c|c|c|c|c|c|}
\hline Disease & Gene & Mutation & Stop codon & Readthrough drug & Application method & Reference \\
\hline PXE & $A B C C 6$ & p.R1141X & UGA & PTC124 & & $(58)$ \\
\hline NPPK & SERPINB7 & c. $796 C>T$ & UGA & Gentamicin & Topical $0.1 \%$ gentamicin & (59) \\
\hline \multirow[t]{3}{*}{ RDEB } & COL7A1 & $\begin{array}{l}\text { R578X/R578X } \\
\text { R613X/R1683X } \\
\text { R578X/V168GfsX12 } \\
\text { R2814X/IVS17-2delA } \\
\text { R236X/IVS85-1G >A }\end{array}$ & & Gentamicin & $\begin{array}{l}\text { Topical } 0.1 \% \text { gentamicin, } \\
\text { intradermal injections }\end{array}$ & $(61)$ \\
\hline & & $\begin{array}{l}\text { p.Q251X } \\
\text { p.R578X/p.Q906X } \\
\text { p.R2610X } \\
\text { p.G2073D/p.R578X }\end{array}$ & $\begin{array}{l}\text { UAG } \\
\text { UGA/UAG } \\
\text { UGA } \\
\text { UGA }\end{array}$ & Gentamicin, amlexanox & & $(62)$ \\
\hline & & $\begin{array}{l}\text { R578X/Q906X } \\
\text { Q251X/Q251X } \\
\text { R578X/R578X } \\
\text { R163X/R1683X }\end{array}$ & $\begin{array}{l}\text { UGA/UAG } \\
\text { UAG }\end{array}$ & Geneticin, paromomycin, gentamicin & & $(63)$ \\
\hline HHD & ATP2C1 & C. $1402 C>T$ & UGA & Gentamicin, paromomycin & Topical $0.1 \%$ gentamicin & $(64)$ \\
\hline \multirow[t]{2}{*}{$X P$} & $X P C$ & & UGA & $\begin{array}{l}\text { Geneticin, gentamicin, PTC124, BZ16, } \\
\text { RTC14 }\end{array}$ & & $(65)$ \\
\hline & $X P A$ & & UGA & Hargsup tRNA ${ }^{\text {opal }}$ & & (5) \\
\hline HSS & $C D S N$ & c. $643 \mathrm{C}>\mathrm{T}$ & UAG & Gentamicin & Topical $0.1 \%$ gentamicin & $(67)$ \\
\hline
\end{tabular}


gene mutations are nonsense mutations, leading to the synthesis of truncated, non-functional ABCC6 protein. A total of 25 different nonsense mutations have been found in the $A B C C 6$ gene of PXE patients, including the most common stop codon mutation, p.R1141X. Cells containing the mutant $A B C C 6$ gene have been treated with different concentrations of PTC124, which can induce the PTC-readthrough in a time-dependent manner. The optimal concentration is $5 \mathrm{mg} / \mathrm{ml}$, and higher concentrations do not improve the readthrough. The efficiency is related to the context sequences of the PTC, which is consistent with previous evidence (58). It is worth noting that PTC124 only causes a partial correction of ABCC6 expression at this concentration, but a small amount of functional protein seems to be sufficient to alleviate the disease phenotype.

\section{Nagashima-type palmoplantar keratosis}

Nagashima-type palmoplantar keratosis (NPPK) is caused by mutations in SERPINB7. Studies have confirmed that gentamicin has a significant readthrough effect in cells transduced with the mutant gene c.796C $>\mathrm{T}$ and in keratinocytes from NPPK patients (59). Subsequently, $0.1 \%$ gentamicin ointment has been externally applied to the affected areas of 5 patients with mutant c.796C $>$ T for 4 weeks, and hyperkeratosis was ameliorated (59). In a recent randomized double-blind controlled clinical trial, 20 NPPK patients with nonsense mutations were given topical gentamicin ointment for 30 days (60). The symptoms of hyperkeratosis and the peculiar smell of the treatment group were significantly improved compared with the control group. The improvement effect of $0.3 \%$ gentamicin ointment was more obvious than that of $0.1 \%$, although there was no statistical difference. However, erythema did not improve significantly, which is consistent with previous studies (60). This may be due to the fact that the level of restored functional protein is still insufficient, or the functional protein induced by gentamicin has not yet fully exerted its effect.

\section{Recessive dystrophic epidermolysis bullosa}

The prevalence of nonsense mutations in recessive dystrophic epidermolysis bullosa (RDEB), in which the production of type VII collagen (COL7A1) is hindered, is close to $30 \%$. A preliminary study involving $0.1 \%$ topical or intradermal injection of gentamicin in 5 RDEB patients with nonsense mutations has provided very encouraging results, clearly indicating the reconstitution of full-length COL7A1 expression and the formation of anchoring fibrils. The frequency of blistering was decreased, and wound healing was accelerated in the experimental group (61). Atanasova et al. (62) demonstrated in in vitro experiments that amlexanox, as a PTC-readthrough compound, induces the synthesis of full-length COL7A1 in fibroblasts and keratinocytes of
RDEB patients. Gentamicin has also been shown to recover functional protein in RDEB cells (both keratinocytes and fibroblasts, which harbour COL7A1 mutation) (63).

\section{Hailey-Hailey disease}

Hailey-Hailey disease (HHD), also known as chronic benign familial pemphigus, is linked to mutations in the $A T P 2 C 1$ gene encoding hSPCA1. More than 80 pathogenic ATP2C1 mutations have been reported in HHD patients, $20 \%$ of which are caused by PTCs resulting in the synthesis of a truncated form of hSPCA1. It has been found that $0.1 \%$ topical gentamicin can reduce the erythema area and increase the healing rate in an HHD patient who carries a UGA nonsense mutation (R468X). In addition, in vitro cell experiments have shown that the full-length hSPCA1 protein is increased in the presence of paromomycin (64).

\section{Xeroderma pigmentosum}

Fifteen percent of patients with xeroderma pigmentosum (XP) have been identified as carrying PTCs. XP is genetically divided into seven different types (XPA to XPG) and one variant (XPV). Studies have reported that treatment of patients' primary XP-C cells with NMD inhibitors can lead to a significant increase in $X P C$ mRNA levels and XPC protein production; the same results can be obtained by treating cell lines with geneticin. Geneticin has higher readthrough efficiency and toxicity than other aminoglycosides, such as gentamicin (10). Treatment with non-aminoglycoside compounds, such as PTC124, BZ16, and RTC14, can also lead to increased levels of XPC mRNA. Although the protein yield is small, as little as $1 \%$ of the normal protein function after PTC-readthrough may be sufficient to restore a nearly normal or clinically less severe phenotype, and the toxicities of these compounds are lower than those of geneticin and gentamicin $(47,65)$. Interestingly, based on the above studies, the authors of one study (65) proposed that topical application of aminoglycoside drugs may also help prevent sun-induced skin cancer in XP patients, and at the same time can greatly reduce the toxic side-effects of the drug.

In another study focusing on XP-A cells in XP disease, suppressor tRNA was found to repair genetic defects caused by nonsense mutations and partially restore the $X P A$ gene deficiency phenotype (5). Perhaps due to the low abundance of XPA transcripts and tRNAs, and multiple factors affecting the readthrough of nonsense suppression and the regulation of other biological mechanisms in the cell, the expression and inhibition efficiency of suppressor tRNAs are still low (66).

\section{Hypotrichosis simplex of the scalp}

Hereditary hypotrichosis simplex of the scalp (HSS) is usually caused by a nonsense mutation in the $C D S N$ gene 
that encodes corneodesmosin. A recent study by Peled et al. (67) has found that gentamicin restores full-length corneodesmosin expression in cells transduced with the mutant gene c.643C $>\mathrm{T}$ and in primary keratinocytes from HSS patients, and local application of $0.1 \%$ gentamicin ointment to the scalp of 4 HSS patients relieved symptoms. This study further confirms the potential value of topical aminoglycoside drugs in the treatment of hereditary skin diseases.

In conclusion, the purpose of nonsense suppression therapy is typically to allow for PTC-readthrough in various ways to hinder the termination of protein translation and ultimately alleviate the disease phenotype. In this review, we introduced the readthrough mechanisms of classic aminoglycosides, the novel compound PTC124, NMD inhibitors, and suppressor tRNA, and summarized the research status of the latest nonsense suppression treatments for hereditary skin diseases. In general, nonsense suppression therapy has achieved positive effects in treating several hereditary skin diseases and may provide a new treatment regimen. But, to date, the application of nonsense suppression therapy in hereditary skin diseases is limited. Emerging technologies such as RNA pseudouridylation, RNA editing, and CRISPR/Cas9 technology have been clearly proven to have therapeutic effects in other non-dermatological genetic diseases (68), but they have not yet been applied to dermatology. Therefore, the above-mentioned technology has broad prospects in the treatment of hereditary skin diseases. In addition, due to the effectiveness of gentamicin topical preparations on skin lesion healing, if aminoglycosides are made into topical preparations, they are very safe under the premise that the blood concentration can be controlled. The development of new dressings or the preparation of other readthrough drugs into topical preparations may also be a direction for future treatments. The combined use of NMD inhibitory compounds and PTC-suppression drugs may improve the effects of nonsense suppression treatment by increasing the abundance of PTC-containing mRNA substrates (33). Some non-aminoglycoside drugs, such as PTC124, also show better human tolerance. The gradual and stable expression of suppressor tRNA will provide advantages for correcting hereditary skin diseases. As a small transcript, tRNA can be developed into nanoparticle materials for topical or systemic delivery. How to maximize the targeting of nonsense suppression therapy and reduce related side-effects will be the focus of future research.

\section{ACKNOWLEDGEMENTS}

This work was supported by the National Natural Science Foundation of China (Number 82003363), the Changsha Municipal Natural Science Foundation (kq2007059) and Hunan Provincial Natural Science Foundation of China (2021JJ40820).

The authors confirm that this study complies with ethical standards.
The authors have no conflicts of interest to declare.

\section{REFERENCES}

1. Porter JJ, Heil CS, Lueck JD. Therapeutic promise of engineered nonsense suppressor tRNAs. Wiley Interdiscip Rev RNA 2021; 12: e1641.

2. Mendell JT, Dietz HC. When the message goes awry: diseaseproducing mutations that influence mRNA content and performance. Cell 2001; 107: 411-414.

3. Baradaran-Heravi A, Balgi AD, Hosseini-Farahabadi S, Choi K, Has C, Roberge M. Effect of small molecule eRF3 degraders on premature termination codon readthrough. Nucleic Acids Res 2021; 49: 3692-3708.

4. Lee HL, Dougherty JP. Pharmaceutical therapies to recode nonsense mutations in inherited diseases. Pharmacol Ther 2012; 136: 227-266.

5. Panchal RG, Wang S, McDermott J, Link CJ. Partial functional correction of xeroderma pigmentosum group A cells by suppressor tRNA. Hum Gene Ther 1999; 10: 2209-2219.

6. Frolova L, Le Goff X, Rasmussen $\mathrm{HH}$, Cheperegin S, Drugeon G, Kress M, et al. A highly conserved eukaryotic protein family possessing properties of polypeptide chain release factor. Nature 1994; 372: 701-703.

7. Temple GF, Dozy AM, Roy KL, Kan YW. Construction of a functional human suppressor tRNA gene: an approach to gene therapy for beta-thalassaemia. Nature 1982; 296: 537-540.

8. Kiselev AV, Ostapenko OV, Rogozhkina EV, Kholod NS, Seit NA, Baranov AN, et al. Suppression of nonsense mutations in the Dystrophin gene by a suppressor tRNA gene. Mol Biol (Mosk) 2002; 36: 43-47.

9. Howard M, Frizzell RA, Bedwell DM. Aminoglycoside antibiotics restore CFTR function by overcoming premature stop mutations. Nat Med 1996; 2: 467-469.

10. Clancy JP, Bebok Z, Ruiz F, King C, Jones J, Walker L, et al. Evidence that systemic gentamicin suppresses premature stop mutations in patients with cystic fibrosis. Am J Respir Crit Care Med 2001; 163: 1683-1692.

11. Howard MT, Shirts BH, Petros LM, Flanigan KM, Gesteland RF, Atkins JF. Sequence specificity of aminoglycoside-induced stop codon readthrough: potential implications for treatment of Duchenne muscular dystrophy. Ann Neurol 2000; 48: 164-169.

12. Sossi V, Giuli A, Vitali T, Tiziano F, Mirabella M, Antonelli A, et al. Premature termination mutations in exon 3 of the SMN1 gene are associated with exon skipping and a relatively mild SMA phenotype. Eur J Hum Genet 2001; 9: 113-120.

13. Kaler SG, Tang J, Donsante A, Kaneski CR. Translational readthrough of a nonsense mutation in ATP7A impacts treatment outcome in Menkes disease. Ann Neurol 2009; 65: 108-113.

14. Lai CH, Chun HH, Nahas SA, Mitui M, Gamo KM, Du L, et al. Correction of ATM gene function by aminoglycoside-induced read-through of premature termination codons. Proc Natl Acad Sci U S A 2004; 101: 15676-15681.

15. Fazzari M, Frasca A, Bifari F, Landsberger N. Aminoglycoside drugs induce efficient read-through of CDKL5 nonsense mutations, slightly restoring its kinase activity. Rna Biol 2019; 16: 1414-1423.

16. Keeling KM, Brooks DA, Hopwood JJ, Li P, Thompson JN, Bedwell DM. Gentamicin-mediated suppression of Hurler syndrome stop mutations restores a low level of alpha-Liduronidase activity and reduces lysosomal glycosaminoglycan accumulation. Hum Mol Genet 2001; 10: 291-299.

17. Helip-Wooley A, Park MA, Lemons RM, Thoene JG. Expression of CTNS alleles: subcellular localization and aminoglycoside correction in vitro. Mol Genet Metab 2002; 75: 128-133.

18. Sangkuhl K, Schulz A, Rompler H, Yun J, Wess J, Schoneberg T. Aminoglycoside-mediated rescue of a disease-causing nonsense mutation in the V2 vasopressin receptor gene in vitro and in vivo. Hum Mol Genet 2004; 13: 893-903.

19. Grayson C, Chapple JP, Willison KR, Webster AR, Hardcastle $A J$, Cheetham ME. In vitro analysis of aminoglycoside therapy for the Arg120stop nonsense mutation in RP2 patients. J Med 
Genet 2002; 39: 62-67.

20. Moosajee M, Tracey-White D, Smart M, Weetall M, Torriano $S$, Kalatzis $V$, et al. Functional rescue of REP1 following treatment with PTC124 and novel derivative PTC-414 in human choroideremia fibroblasts and the nonsense-mediated zebrafish model. Hum Mol Genet 2016; 25: 3416-3431.

21. Moosajee M, Gregory-Evans K, Ellis CD, Seabra MC, GregoryEvans CY. Translational bypass of nonsense mutations in zebrafish rep1, pax2.1 and lamb1 highlights a viable therapeutic option for untreatable genetic eye disease. Hum Mol Genet 2008; 17: 3987-4000.

22. Rebibo-Sabbah A, Nudelman I, Ahmed ZM, Baasov T, BenYosef T. In vitro and ex vivo suppression by aminoglycosides of PCDH15 nonsense mutations underlying type 1 Usher syndrome. Hum Genet 2007; 122: 373-381.

23. Nishimoto K, Iijima K, Shirakawa T, Kitagawa K, Satomura $\mathrm{K}$, Nakamura $\mathrm{H}$, et al. PAX2 gene mutation in a family with isolated renal hypoplasia. J Am Soc Nephrol 2001; 12: 1769-1772.

24. Richardson R, Smart M, Tracey-White D, Webster AR, Moosajee $M$. Mechanism and evidence of nonsense suppression therapy for genetic eye disorders. Exp Eye Res 2017; 155: 24-37.

25. Isidor B, Lindenbaum P, Pichon O, Bezieau S, Dina C, Jacquemont $S$, et al. Truncating mutations in the last exon of NOTCH2 cause a rare skeletal disorder with osteoporosis. Nat Genet 2011; 43: 306-308.

26. Castaman G, Bertoncello K, Bernardi M, Eikenboom JC, Budde U, Rodeghiero F. Autosomal recessive von Willebrand disease associated with compound heterozygosity for a novel nonsense mutation ( $2908 \mathrm{del} \mathrm{C}$ ) and the missense mutation C2362F: definite evidence for the non-penetrance of the C2362F mutation. Am J Hematol 2007; 82: 376-380.

27. Bezzerri V, Cipolli M. Shwachman-Diamond syndrome: molecular mechanisms and current perspectives. Mol Diagn Ther 2019; 23: 281-290.

28. Dorin JR, Farley R, Webb S, Smith SN, Farini E, Delaney SJ, et al. A demonstration using mouse models that successful gene therapy for cystic fibrosis requires only partial gene correction. Gene Ther 1996; 3: 797-801.

29. Krause KM, Serio AW, Kane TR, Connolly LE. Aminoglycosides: an overview. Cold Spring Harb Perspect Med 2016; 6: a027029.

30. Keeling KM, Wang D, Conard SE, Bedwell DM. Suppression of premature termination codons as a therapeutic approach. Crit Rev Biochem Mol Biol 2012; 47: 444-463.

31. Schueren F, Thoms S. Functional translational readthrough: a systems biology perspective. Plos Genet 2016; 12: e1006196.

32. Leier A, Bedwell DM, Chen AT, Dickson G, Keeling KM, Kesterson RA, et al. Mutation-directed therapeutics for neurofibromatosis Type I. Mol Ther Nucleic Acids 2020; 20: 739-753.

33. McHugh DR, Cotton CU, Hodges CA. Synergy between readthrough and nonsense mediated decay inhibition in a murine model of cystic fibrosis nonsense mutations. Int J Mol Sci 2020; 22: 344.

34. Prokhorova I, Altman RB, Djumagulov M, Shrestha JP, Urzhumtsev A, Ferguson A, et al. Aminoglycoside interactions and impacts on the eukaryotic ribosome. Proc Natl Acad Sci U S A 2017; 114: e10899-e10908.

35. Baradaran-Heravi A, Niesser J, Balgi AD, Choi K, Zimmerman C, South AP, et al. Gentamicin B1 is a minor gentamicin component with major nonsense mutation suppression activity. Proc Natl Acad Sci U S A 2017; 114: 3479-3484.

36. Pasmooij A. Topical gentamicin for the treatment of genetic skin diseases. J Invest Dermatol 2018; 138: 731-734.

37. Linde $L$, Kerem $B$. Introducing sense into nonsense in treatments of human genetic diseases. Trends Genet 2008; 24: 552-563.

38. Dabrowski M, Bukowy-Bieryllo Z, Zietkiewicz E. Advances in therapeutic use of a drug-stimulated translational readthrough of premature termination codons. Mol Med 2018; 24: 25.

39. Nudelman I, Rebibo-Sabbah A, Cherniavsky M, Belakhov $V$, Hainrichson $M$, Chen $F$, et al. Development of novel ami- noglycoside (NB54) with reduced toxicity and enhanced suppression of disease-causing premature stop mutations. J Med Chem 2009; 52: 2836-2845.

40. Hainrichson M, Nudelman I, Baasov T. Designer aminoglycosides: the race to develop improved antibiotics and compounds for the treatment of human genetic diseases. Org Biomol Chem 2008; 6: 227-239.

41. Hirawat S, Welch EM, Elfring GL, Northcutt VJ, Paushkin S, Hwang S, et al. Safety, tolerability, and pharmacokinetics of PTC124, a nonaminoglycoside nonsense mutation suppressor, following single- and multiple-dose administration to healthy male and female adult volunteers. J Clin Pharmacol 2007; 47: 430-444.

42. Campofelice A, Lentini L, Di Leonardo A, Melfi R, Tutone M, Pace $A$, et al. Strategies against nonsense: oxadiazoles as translational readthrough-inducing drugs (TRIDs). Int J Mol Sci 2019; 20: 3329.

43. Du M, Liu X, Welch EM, Hirawat S, Peltz SW, Bedwell DM. PTC124 is an orally bioavailable compound that promotes suppression of the human CFTR-G542X nonsense allele in a CF mouse model. Proc Natl Acad Sci U S A 2008; 105: 2064-2069.

44. Lentini L, Melfi R, Di Leonardo A, Spinello A, Barone G, Pace A, et al. Toward a rationale for the PTC124 (Ataluren) promoted readthrough of premature stop codons: a computational approach and GFP-reporter cell-based assay. Mol Pharm 2014; 11: 653-664.

45. Roy B, Friesen WJ, Tomizawa Y, Leszyk JD, Zhuo J, Johnson $B$, et al. Ataluren stimulates ribosomal selection of nearcognate tRNAs to promote nonsense suppression. Proc Natl Acad Sci U S A 2016; 113: 12508-12513.

46. McElroy SP, Nomura T, Torrie LS, Warbrick E, Gartner U, Wood $\mathrm{G}$, et al. A lack of premature termination codon read-through efficacy of PTC124 (Ataluren) in a diverse array of reporter assays. Plos Biol 2013; 11: e1001593.

47. Du L, Damoiseaux R, Nahas S, Gao K, Hu H, Pollard JM, et al. Nonaminoglycoside compounds induce readthrough of nonsense mutations. J Exp Med 2009; 206: 2285-2297.

48. Kayali R, Ku JM, Khitrov G, Jung ME, Prikhodko O, Bertoni C. Read-through compound 13 restores dystrophin expression and improves muscle function in the mdx mouse model for Duchenne muscular dystrophy. Hum Mol Genet 2012; 21: 4007-4020.

49. Du L, Jung ME, Damoiseaux R, Completo G, Fike F, Ku JM, et al. A new series of small molecular weight compounds induce read through of all three types of nonsense mutations in the ATM gene. Mol Ther 2013; 21: 1653-1660.

50. Friesen WJ, Trotta CR, Tomizawa Y, Zhuo J, Johnson B, Sierra $\mathrm{J}$, et al. The nucleoside analog clitocine is a potent and efficacious readthrough agent. RNA 2017; 23: 567-577.

51. Arakawa M, Shiozuka M, Nakayama Y, Hara T, Hamada M, Kondo $S$, et al. Negamycin restores dystrophin expression in skeletal and cardiac muscles of mdx mice. J Biochem 2003; 134: 751-758.

52. Thompson J, Pratt CA, Dahlberg AE. Effects of a number of classes of 505 inhibitors on stop codon readthrough during protein synthesis. Antimicrob Agents Chemother 2004; 48: 4889-4891.

53. Lykke-Andersen S, Jensen TH. Nonsense-mediated mRNA decay: an intricate machinery that shapes transcriptomes. Nat Rev Mol Cell Biol 2015; 16: 665-677.

54. Brogna S, Wen J. Nonsense-mediated mRNA decay (NMD) mechanisms. Nat Struct Mol Biol 2009; 16: 107-113.

55. Azzalin CM, Lingner J. The human RNA surveillance factor UPF1 is required for $S$ phase progression and genome stability. Curr Biol 2006; 16: 433-439.

56. Lueck JD, Yoon JS, Perales-Puchalt A, Mackey AL, Infield DT, Behlke MA, et al. Engineered transfer RNAs for suppression of premature termination codons. Nat Commun 2019; 10: 822.

57. Buvoli M, Buvoli A, Leinwand LA. Suppression of nonsense mutations in cell culture and mice by multimerized suppressor tRNA genes. Mol Cell Biol 2000; 20: 3116-3124.

58. Zhou Y, Jiang Q, Takahagi S, Shao C, Uitto J. Premature termination codon read-through in the ABCC6 gene: potential treatment for pseudoxanthoma elasticum. J Invest Dermatol 
2013; 133: 2672-2677.

59. Ohguchi Y, Nomura T, Suzuki S, Takeda M, Miyauchi T, Mizuno $O$, et al. Gentamicin-induced readthrough and nonsensemediated mRNA decay of SERPINB7 nonsense mutant transcripts. J Invest Dermatol 2018; 138: 836-843.

60. Li Y, Yu X, Pan C, Wang Y, Han J, Yao Z, et al. Effect of gentamicin ointment in patients with nagashima-type palmoplantar keratosis: a double-blind vehicle-controlled study. Acta Derm Venereol 2021; 101: adv00392.

61. Woodley DT, Cogan J, Hou Y, Lyu C, Marinkovich MP, Keene $D$, et al. Gentamicin induces functional type VII collagen in recessive dystrophic epidermolysis bullosa patients. J Clin Invest 2017; 127: 3028-3038.

62. Atanasova VS, Jiang Q, Prisco M, Gruber C, Pinon HJ, Chen $M$, et al. Amlexanox enhances premature termination codon read-through in COL7A1 and expression of full length Type VII collagen: potential therapy for recessive dystrophic epidermolysis bullosa. J Invest Dermatol 2017; 137: 1842-1849.

63. Cogan J, Weinstein J, Wang X, Hou Y, Martin S, South AP, et al. Aminoglycosides restore full-length type VII collagen by overcoming premature termination codons: therapeutic implications for dystrophic epidermolysis bullosa. Mol Ther 2014; 22: 1741-1752.

64. Kellermayer R, Szigeti R, Keeling KM, Bedekovics T, Bedwell DM. Aminoglycosides as potential pharmacogenetic agents in the treatment of Hailey-Hailey disease. J Invest Dermatol 2006; 126: 229-231.

65. Kuschal C, DiGiovanna JJ, Khan SG, Gatti RA, Kraemer KH. Repair of UV photolesions in xeroderma pigmentosum group $\mathrm{C}$ cells induced by translational readthrough of premature termination codons. Proc Natl Acad Sci U S A 2013; 110: 19483-19488.

66. Satokata I, Tanaka K, Okada Y. Molecular basis of group A xeroderma pigmentosum: a missense mutation and two deletions located in a zinc finger consensus sequence of the XPAC gene. Hum Genet 1992; 88: 603-607.

67. Peled A, Samuelov L, Sarig O, Bochner R, Malki L, Pavlovsky $M$, et al. Treatment of hereditary hypotrichosis simplex of the scalp with topical gentamicin. Br J Dermatol 2020; 183: 114-120.

68. Morais P, Adachi $H$, Yu YT. Suppression of nonsense mutations by new emerging technologies. Int J Mol Sci 2020; 21: 4394. 